\section{An overseas trainee's experience at the College annual conference, London, 2008}

As an invited speaker from the Western Pacific
Division of the Board of International Affairs, and as a third-year trainee from Hong Kong, I attended the College annual conference in London, 1-4 July 2008. It was truly an eye-opening experience and I took home with me many wonderful memories and invaluable knowledge.

During the four days of the conference, I met eminent professors, respected psychiatrists, specialists and trainees from all over the world. I attended meetings of the Board of International Affairs, where many issues were raised, ranging from disaster management to organising training programmes and various campaigns. This was an area of psychiatry that I had not been exposed to before, and it was an introduction to what psychiatry on a global scale entails.

As for my presentation, the title was 'Getting it across: psycho-education in primary care'. There was a lively question-and-answer session afterwards, and the feedback was positive and encouraging.

Last but not least, the conference offered a myriad of symposiums and talks to suit everyone's specialties and particular interests. The presentations were done in a very personal and interactive manner, which made learning much more interesting than the standard lectures.

As for social events, I attended the trainees' reception and met others from different levels of training in the UK. It gave me an opportunity to reflect on the situation in Hong Kong, and to learn about the changes and current situation in UK training as well. The highlight of the week was the cocktail reception at the House of Lords for overseas delegates. After a welcoming speech from Lord Crisp, who has been involved in mental healthcare issues for many years, we were given a guided tour around the famous parts of Parliament: the House of Lords, House of Commons and various other rooms and halls. It was an unforgettable evening spent in the company of many great people.

I realised that a career in psychiatry is more than just passing exams, getting a training post, or even publishing papers. There are so many ways in which we, even as trainees and younger members of the psychiatric profession, can serve our community. Getting into volunteer programmes, raising money for low-income countries, coordinating response protocols for disaster-stricken areas, and advancing psychiatric training and education globally are but a few ways in which we can get help, and the College provides a platform for us to participate in such activities. The satisfaction and personal experiences I gained from this conference gave me motivation to be a better psychiatrist, and provided me with a sense of direction in my professional career.

Vanessa T. C. Wong Resident, psychiatrist trainee, Hong Kong, emailvtcwong@gmail.com

\section{Sensitising primary care physicians to common mental disorders using a problem-based learning approach}

C. We conducted a series of 1-day sensitisation - workshops on common mental disorders for primary care physicians in Delhi. The workshops were conducted as part of the National Mental Health Programme of the government of India. The workshops were kept to 1 day because of the busy clinical schedules of the medical officers. The learning objectives were that the medical officers would be able to diagnose and manage these disorders in their clinical practice using both pharmacological and basic non-pharmacological interventions, such as reassurance, encouragement and psychoeducation.

The programme consisted of three sessions, one each on anxiety disorders, depressive disorders and somatoform disorders. These conditions were chosen because they are common, often remain undetected, are associated with significant dysfunction and medical comorbidity, and are easy to treat in primary care. Three workshops were conducted and 102 primary care physicians attended. To encourage the active involvement of participants, a problem-based learning approach was used (Wood, 2003).

The participants were divided into groups of six to eight. Each session comprised a group task, presentation of the problem solution by one of the group members and a brief discussion. Each group was given a problem in the form of a case vignette along with five questions related to diagnosis and management. The groups were given 10 minutes for the task followed by 5 minutes for presentation and discussion. This was followed by an interactive presentation on the topic by one of the psychiatrists, followed by general discussion.

At the end of the presentation, 10 minutes were given for feedback from administrators and participants. The programme format was found to be very useful by most people, as it had made them participate in the learning, and they had an opportunity to discuss the difficulties of dealing with such cases during day-to-day clinical practice.

Under the National Mental Health Programme, 2-week training programmes have been conducted in different places in India and have been found useful in improving primary care workers' knowledge of mental health issues (Sriram et al, 1990). But it has often been found difficult for doctors working in primary care to be sent for 2 weeks' training. Most of the earlier programmes have been in the form of didactic lectures. The present programme in the form of interactive sessions lasting just 1 day can serve as a useful training strategy for use in low- and middle-income countries, where there is a major shortage of health personnel.

Sriram, T. G., Chandrashekar, C. R., Isaac, M. K., et al (1990) Development of case vignettes to assess the mental health training of primary care medical officers. Acta Psychiatrica Scandinavica, 82, 174-177.

Wood, D. F. (2003) ABC of learning and teaching in medicine: problem based learning. BMJ, 326, 328-330.

Rakesh K. Chadda, Mamta Sood and Nand Kumar Department of Psychiatry, All India Institute of Medical Sciences, Ansari Nagar, New Delhi 110029, India, email drrakeshchadda@hotmail.com 